\title{
A successful obstetric outcome in pregnancy complicated with cor pulmonale, pulmonary hypertension secondary to pulmonary tuberculosis: a case report
}

\author{
Rao Preethi Venkatachala ${ }^{1 *}$, C. N. Sheela ${ }^{1}$, Shobha G. ${ }^{1}$, Uma Devaraj ${ }^{2}$, \\ Arpana Kedlaya ${ }^{3}$, Kiron Varghese ${ }^{4}$
}

\begin{abstract}
${ }^{1}$ Department of Obstetrics and Gynaecology, ${ }^{2}$ Department of Pulmonology, ${ }^{3}$ Department of Anaesthesia, ${ }^{4}$ Department of Cardiology, St Johns Medical College and Hospital, Bengaluru, Karnataka, India
\end{abstract}

Received: 12 May 2018

Accepted: 05 June 2018

\section{*Correspondence:}

Dr. Rao Preethi Venkatachala,

E-mail: preethibhat77@gmail.com

Copyright: () the author(s), publisher and licensee Medip Academy. This is an open-access article distributed under the terms of the Creative Commons Attribution Non-Commercial License, which permits unrestricted non-commercial use, distribution, and reproduction in any medium, provided the original work is properly cited.

\begin{abstract}
Pregnancy in patients with pulmonary hypertension and cor pulmonale is associated with high morbidity and mortality. The timing of termination of pregnancy, mode of delivery, labour analgesia and anaesthesia and management during puerperium are challenging issues which need to be resolved. Authors report a case of a 30-yearold primigravida with cor pulmonale and severe pulmonary artery hypertension secondary to past pulmonary tuberculosis, who delivered a healthy baby vaginally, due to its rarity and good outcome. Early diagnosis, effective management of aggravating factors like anaemia and hypertension, informed choice by the couple regarding route of delivery and multidisciplinary approach are the keys to successful outcomes in patients with cor pulmonale.
\end{abstract}

Keywords: Cor pulmonale, Pregnancy outcome, Pulmonary hypertension, Tuberculosis

\section{INTRODUCTION}

Pulmonary hypertension $(\mathrm{PH})$ is defined as an increase in mean pulmonary arterial pressure $(\mathrm{mPAP}) \geq 25 \mathrm{mmHg}$ at rest as assessed by right heart catheterisation. ${ }^{1}$

It is characterized by progressive dyspnoea, exercise intolerance, and right heart failure, leading eventually to death. Median survival was reported to be 2.8 years. $^{2}$

Pulmonary hypertension is clinically classified into 5 groups:

- group 1 includes PH which may be idiopathic, heritable, induced by drugs or toxins or associated with connective tissue disorders, HIV infection, portal hypertension or congenital heart disease

- group 2 consists of PH due to left heart disease
- group 3 includes PH due to lung diseases and/or hypoxia (such as chronic obstructive pulmonary disease, interstitial lung disease and others)

- $\quad$ group 4 includes chronic thrombo embolic PH and

- group 5 consists of $\mathrm{PH}$ with unclear and/or multifactorial mechanisms. ${ }^{1}$

Management of pregnancies in patients with pulmonary hypertension secondary to lung diseases poses a great challenge to the medical fraternity. The high rates of maternal and foetal morbidity and mortality reported due to $\mathrm{PH}$ are a deterrent to pregnancy. ${ }^{3}$ Physiologic changes in pregnancy may delay the diagnosis. Several practice guidelines advise against pregnancy and even recommend termination of pregnancy. ${ }^{4}$ The timing of termination, mode of delivery, type of analgesia and anaesthesia and methods to reduce the risk in puerperal period are burning issues which need to be answered. This case is 
being reported due to its rarity and good maternal and foetal outcome.

\section{CASE REPORT}

A 30-year-old primigravida with persistent breathlessness and respiratory failure was referred to us for further management. Her pregnancy was uneventful till she developed breathlessness at 23 weeks. She gave history of pulmonary tuberculosis (TB) 15 years ago, when she took anti tuberculous medicines for 6 months. As her electrocardiogram was normal and $\mathrm{H} 1 \mathrm{~N} 1$ and $\mathrm{TB}$ work up were negative (Figure 1), she was conservatively managed with oxygen and salbutamol nebulisation at a hospital elsewhere.

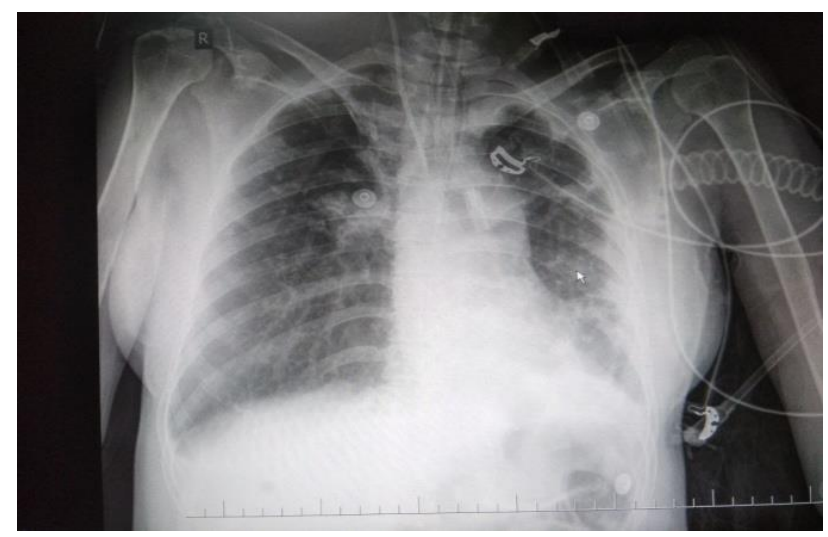

Figure 1: Chest $X$ ray: fibrosis of left lung; right, middle and lower zone fibrotic bands.

The patient was readmitted for breathlessness in another hospital at 25 weeks, where an echocardiography was done which revealed severe PH with pulmonary artery systolic pressure of $91 \mathrm{~mm} \mathrm{Hg}$, severe tricuspid regurgitation but adequate left ventricular systolic function with left ventricular ejection fraction of $53 \%$. Moderate anaemia $(\mathrm{Hb}-8 \mathrm{~g} / \mathrm{dl})$ was corrected with parenteral iron therapy. At 28 weeks, CT Pulmonary Angiography (Figure 2) was done in view of persistent orthopnoea which confirmed the diagnosis of $\mathrm{PH}$ with extensive fibrosis in both lungs as a sequela of pulmonary TB, without features of thromboembolism. A trial of Digoxin and Sildenafil for 1 week did not yield much symptomatic improvement. Hence the patient was referred to the present hospital.

On admission to the present hospital at 28 weeks, she had a pulse rate of $128 \mathrm{bpm}$, blood pressure $-100 / 70 \mathrm{~mm} \mathrm{Hg}$, $\mathrm{SaO} 2-70-80$, raised jugular venous pressure, loud pulmonary component of second heart sound and scattered crackles in both lungs. A diagnosis of bilateral pulmonary fibrosis secondary to past pulmonary TB with type 1 respiratory failure and severe pulmonary hypertension complicated with lower respiratory tract infection was made. She was managed conservatively by the pulmonologist with supplemental oxygen, antibiotics and salbutamol nebulisation. Digoxin and Sildenafil were withdrawn, and she was discharged with advice to continue domiciliary oxygen.

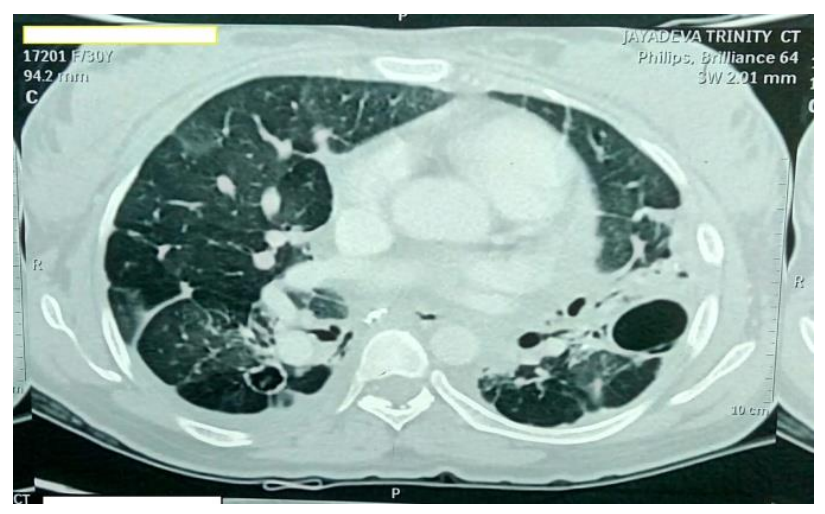

Figure 2: CT chest: left lingular cavitation and fibrosis- bilateral mosaic pattern and fibrotic bands seen.

The patient was seen by the Obstetric team on an outpatient basis every 2 week, as her blood pressure and blood sugars were normal, anaemia corrected $(\mathrm{Hb}-$ $11 \mathrm{~g} / \mathrm{dl}$ ) and there was no intrauterine growth restriction. Her anomaly ultrasound (USG) and growth USG at 34 weeks were normal. She was readmitted at 35 weeks under the pulmonologist for hemoptysis which was conservatively managed with oxygen and fluid restriction to $<1.5$ Litre/day and subsequently discharged.

She was admitted for safe confinement by the Obstetrics team at 37 weeks; kept on bed rest, continuous oxygen therapy, propped up position, nutritional supplements and salbutamol nebulisation. Her vital signs and foetal heart sounds were monitored. Serial non-stress tests done were reactive. A multidisciplinary team comprising of the Cardiologist, Pulmonologist, Anaesthetist, Intensivist and Neonatologist were involved in the patient care and delivery was planned at 39 weeks. High risk consent was obtained from the couple after explaining the risks and benefits of vaginal delivery versus a Caesarean delivery.

She went into labour spontaneously at $38+6$ weeks. With the onset of true labour pains, she was shifted to intensive care unit (ICU) where strict monitoring of her vital signs and fluid input and output was done. She was kept on clear fluids throughout labour. Labour was monitored using a partogram. An epidural catheter was inserted by the anaesthetist at $2 \mathrm{~cm}$ dilatation to facilitate labour analgesia using $0.0625 \%$ Bupivacaine and $2 \mu \mathrm{g} / \mathrm{ml}$ Fentanyl infusion at $3-4 \mathrm{ml} /$ hour. She leaked at $3 \mathrm{~cm}$ dilatation and was detected to have grade3 meconium stained liquor. Patient and her husband refused an offer of Caesarean Section for foetal distress. She became fully dilated in 7 hours and delivered a healthy baby boy weighing $2.25 \mathrm{~kg}$ with Apgar 1'-8/10 and 5'- 8/10. Low cavity forceps was used to cut short 2 nd stage to avoid maternal effort. Injection Frusemide was given soon after the delivery of the baby. Active management of 3rd stage of labour was done. Mild atonic postpartum haemorrhage 
(PPH) was managed with PGE1 $800 \mathrm{mcg}$ per rectum, concentrated Oxytocin drip of $20 \mathrm{U}$ in $500 \mathrm{ml}$ Ringers Lactate and continuous uterine massage.

Two hours after vaginal delivery, patient developed tachycardia, tachypnoea, hypotension and Pulmonary oedema. She was intubated and connected to IPPV mode of ventilation with $\mathrm{FiO} 2$ of $40 \%$. Nor Adrenaline drip was started to maintain blood pressures and delayed weaning off the ventilator planned. Patient was extubated after 18 hours and was stable on Oxygen 2L using nasal prongs. Gradually, nor adrenaline drip was tapered and stopped, and patient encouraged to take orally with strict input output charting. She was shifted back to ward on postnatal day 2 where thromboprophylaxis was continued. She breastfed her baby and was discharged on postnatal day 7 on oxygen and haematinics.

\section{DISCUSSION}

Pulmonary hypertension in pregnancy is a high-risk pregnancy which needs multidisciplinary approach to improve maternal and neonatal outcome. ${ }^{5}$ With TB being a major health hazard in India and technical advances in diagnosis, more cases of secondary $\mathrm{PH}$ with cor pulmonale may be detected.

Weiss et al have reported that the outcome of patients with secondary $\mathrm{PH}$ is worse than primary $\mathrm{PH}$ and Eisenmenger syndrome (maternal mortality $56 \%$ versus $30 \%$ and $36 \%) .{ }^{3}$ Hence, early detection of these cases and timely termination of pregnancy is essential. Foetal or neonatal death rate is $9.6 \% .^{6}$ Deterioration of the medical condition is reported between $20-24$ weeks, early third trimester and postpartum. ${ }^{7}$

Supplemental oxygen is one of the most effective drugs in lowering pulmonary artery pressures. ${ }^{8}$ Though sildenafil is found to be useful in $\mathrm{PH}$ secondary to heart disease it may not be beneficial in PH secondary to lung disease. ${ }^{7,9}$ Correction of anaemia and prevention of systemic hypertension help in better cardiac performance during delivery. Frequently, delivery is preterm either due to maternal worsening or planned delivery at 34 36weeks gestation. ${ }^{5,6,9}$

Although some authors advocate Caesarean delivery due to day time scheduling and avoidance of prolonged labour and marked haemodynamic changes that may occur with vaginal delivery, no clear survival advantage was noted with any particular mode of delivery. $4,6,7$ Although cardiac output increases by up to $25 \%$ in active labour and $50 \%$ during maternal pushing efforts, assisted $2^{\text {nd }}$ stage labour and labouring in lateral decubitus position can greatly offset these changes. ${ }^{10}$

Cardiac output in postpartum period can increase by $80 \%$ regardless of delivery route. Induction of regional anaesthesia for Caesarean delivery can result in hypotension in upto $30 \%$ of cases. ${ }^{10}$
Low epidural dose with slow titration minimises the haemodynamic compromise associated with systemic afterload reduction. ${ }^{11}$ With the overall mortality of $36 \%$, assisted vaginal delivery with epidural analgesia may be safer and better tolerated by the patient. Caesarean sections must be reserved for obstetrics indications only. ${ }^{8,10}$ As risk of maternal mortality is high during puerperium, vigilance in critical care setup may be required after the delivery. 5,7

Present patient had worsening of maternal condition at 23 weeks and repeated admissions for evaluation and diagnosis. Sildenafil was withheld after consultation with the pulmonologist. Caesarean section, with its major risk of difficulty in weaning off the ventilator, postoperative pulmonary infections, thromboembolism, higher rates of maternal deaths, haemorrhage, shock, cardiac arrest and renal failure, was avoided.

As epidural analgesia was given, drugs that depress respiration or aggravate $\mathrm{PH}$ (e.g. narcotics, ergot alkaloids) were avoided. She responded well to management and gave birth to a healthy baby despite the severity of her lung disease, cor pulmonale and severe PH. A multidisciplinary approach with a team of specialists readily available, round the clock, in a tertiary care centre is crucial for a good outcome.

\section{CONCLUSION}

Early diagnosis, effective management of aggravating factors like anaemia and hypertension, informed choice by the couple regarding route of delivery and multidisciplinary approach are the keys to successful outcomes in patients with cor pulmonale.

Funding: No funding sources

Conflict of interest: None declared

Ethical approval: Not required

\section{REFERENCES}

1. Galiè N, Hoeper MM, Humbert M, Torbicki A, Vachiery JL, Barbera JA, et al. Guidelines for the diagnosis and treatment of pulmonary hypertension: the Task Force for the Diagnosis and Treatment of Pulmonary Hypertension of the European Society of Cardiology (ESC) and the European Respiratory Society (ERS), endorsed by the International Society of Heart and Lung Transplantation (ISHLT). Europ Heart J. 2009 Aug;30(20):2493-537.

2. Seckel MA, Gray C, Farraj MB, Obrien G. Undiagnosed pulmonary arterial hypertension at 33 weeks' gestation: A case report. Crit Care Nurse. 2010;30:45-52.

3. Weiss BM, Zemp L, Siefert B, Hess OM. Outcome of pulmonary vascular disease in pregnancy: a systematic overview from 1978 through 1996. J Am Coll Cardiol .1998;31:1650-7. 
4. Khan J, Idrees MM. Saudi Guidelines on the Diagnosis and Treatment of Pulmonary Hypertension: Pregnancy in pulmonary hypertension. Annals of thoracic medicine. 2014 Jul;9(Suppl 1):S108.

5. Kiely DG, Condliffe R, Webster V, Mills GH, Wrench I, Gandhi SV et al. Improved survival in pregnancy and pulmonary hypertension using a multiprofessional approach. BJOG. 2010 Apr;117(5):565-74.

6. Bédard E, Dimopoulos K, Gatzoulis MA. Has there been any progress made on pregnancy outcomes among women with pulmonary arterial hypertension? Eur Heart J. 2009Feb;30(3):256-65.

7. Hemnes AR, Kiely DG, Cockrill BA, Safdar Z, Wilson VJ, Hazmi MA, et al. Statement on pregnancy in pulmonary hypertension from the Pulmonary Vascular Research Institute. Pulmonary circulation. 2015 Sep;5(3):435-65.

8. Al-Mobeireek AF, Almutawa J, Alsatli RA. The nineteenth pregnancy in a patient with corpulmonale and severe pulmonary hypertension: a management challenge. Acta Obstet Gynecol Scand. 2003 Jul; 82(7):676-8.
9. Sun X, Wang K, Wang W, Li B. Clinical study on sildenafil in treatment of pregnant women with pulmonary arterial hypertension. Zhonghua Fu Chan Ke Za Zhi. 2014 Jun;49(6):414-8.

10. Sharma K, Afshar YR, Bairey-Merz CN, Tapson V, Zakowski M, Kilpatrick SJ. Guidelines and consensus: statement on pregnancy in pulmonary hypertension from the Pulmonary Vascular Research Institute. Pulmonary circulation. 2016 Mar;6(1):143.

11. Tonelli AR, Minai OA. Saudi Guidelines on the Diagnosis and Treatment of Pulmonary Hypertension: Perioperative management in patients with pulmonary hypertension. Ann Thorac Med. 2014 Jul;9(Suppl 1):S98-S107.

Cite this article as: Venkatachala RP, Sheela CN, G Shobha, Devaraj U, Kedlaya A, Varghese K. A successful obstetric outcome in pregnancy complicated with cor pulmonale, pulmonary hypertension secondary to pulmonary tuberculosis: a case report. Int J Reprod Contracept Obstet Gynecol 2018;7:2975-8. 